\title{
Außerschulische Interessen als Ressource für die individualisierte Vermittlung der Variablenkontrollstrategie in der Grundschule
}

\author{
Ann-Kathrin Laufs $(\mathbb{D} \cdot$ Sebastian Kempert $\mathbb{D}$
}

Eingegangen: 14. August 2020 / Überarbeitet: 27. Dezember 2020 / Angenommen: 29. Dezember 2020 / Online publiziert: 18. Januar 2021

(C) Der/die Autor(en) 2021

Zusammenfassung Interesse stellt eine günstige Ausgangsbedingung für Lernprozesse dar. Lernförderliche Effekte des Interessenseinbezugs, wie eine erhöhte Aufmerksamkeit und ein erhöhtes Engagement, sind für die Altersgruppe der Sekundarschülerinnen und -schüler in unterschiedlichen Domänen gut belegt. Dieser Beitrag hat zum Ziel, den Forschungsstand zum Zusammenhang von Interesse und Lernen um die Altersgruppe der Grundschulkinder im Inhaltsbereich des frühen naturwissenschaftlichen Sachunterrichts zu ergänzen. An $N=162$ Grundschulkindern der dritten und vierten Jahrgangsstufe wird im Rahmen einer experimentellen Interventionsstudie untersucht, ob eine an individuellen Interessen ausgerichtete und so kontextualisierte Vermittlung der Variablenkontrollstrategie (VKS) positive Effekte auf (1) das situationale Interesse am Lernkontext und am Lerngegenstand und (2) auf den Lernerfolg hat. Darüber hinaus wird überprüft, (3) ob die angenommenen positiven Effekte einer kontextualisierten Vermittlung auf den Lernerfolg über das situationale Interesse in der Lernsituation vermittelt werden.

Die Ergebnisse belegen eine erfolgreiche Manipulation des situationalen Interesses am Lernkontext und am Lerngegenstand durch die kontextualisierte Vermittlung. Multivariate Analysen weisen ein erhöhtes situationales Interesse am Lernkontext und am Lerngegenstand der Experimentalgruppe 1 gegenüber der Experimentalgruppe 2 und der Kontrollgruppe nach. Differenzierte Analysen zum Lernerfolg der VKS zeigen, dass die an individuellen Interessen ausgerichtete Vermittlung in der Experimentalgruppe 1 zwar zu einem signifikanten Vorteil gegenüber der Kontrollgruppe, jedoch lediglich zu einem deskriptiven Vorteil gegenüber der Experimentalgruppe 2 führt. In einem Mediationsmodell zeigen sich entgegengesetzte Effekte des Interesses am Lernkontext und des Interesses am Lerngegenstand auf die Posttestleistung.

A.-K. Laufs $(\square) \cdot$ S. Kempert

Strukturbereich Bildungswissenschaften, Universität Potsdam, Potsdam, Deutschland

E-Mail: laufs@uni-potsdam.de 
Die Befunde werden vor dem Hintergrund der hohen Bedeutung von individualisierenden didaktischen Konzepten im Grundschulunterricht diskutiert.

Schlüsselwörter Interesse · Aufgabenkontextualisierung · Individualisierung · Variablenkontrollstrategie

\title{
Extracurricular interests as a resource for the individualized teaching of the control-of-variables strategy in primary school
}

\begin{abstract}
Individual interest is considered as a condition for successful learning processes. The effects of interest on learning, such as increased attention and increased commitment are well documented for the group of secondary school students in different domains. This paper aims to complement the current state of research on interest and learning with regard to the group of primary school students in the content area of early science education. In an experimental intervention study we investigate with $N=162$ third-and-fourth-graders whether teaching the control-ofvariables strategy (CVS) contextualized in individual interests has positive effects on (1) their situational interest in the learning context and the learning content and (2) on the learning outcome. Furthermore, we examined (3) whether the assumed positive effects of interest-based tasks on comprehension of the CVS are mediated by children's situational interest in the learning situation.

The results prove a successful manipulation of children's situational interest in the learning context and the learning content. Multivariate analyses demonstrate an increased situational interest in the learning context and the learning content in the experimental group 1 compared to the experimental group 2 and the control group. Differential analyses of the learning outcome on comprehension of the CVS show that interest-based tasks in the experimental group 1 lead to a significant advantage over the control group but only to a descriptive advantage over the experimental group 2. In a mediation model, opposite effects of interest in the learning context and interest in the learning content on post-test performance are shown. The findings are discussed in the context of the high importance of individualizing didactic concepts in primary school.
\end{abstract}

Keywords Interest · Task Context · Individualization · Control-of-Variables Strategy

\section{Einleitung}

Im 21. Jahrhundert sieht sich die Wissensgesellschaft mit vielfachen Herausforderungen in der Verarbeitung und Bewertung von Informationen konfrontiert (Zachmann und Ehlers 2019). Vor diesem Hintergrund erscheint es besonders wichtig, Kompetenzen wissenschaftlichen Denkens, wie Informationen zu beurteilen und neues, belastbares Wissen zu generieren, angemessen zu fördern und somit einen hohen Grad an gesellschaftlicher Partizipation zu ermöglichen (Fischer et al. 2014). Der naturwissenschaftliche Sachunterricht bietet den schulischen Rahmen für die Ent- 
wicklung dieser Kompetenzen in der Primarstufe (z. B. Rahmenlehrplan 1-10 Berlin und Brandenburg, Berliner Senatsverwaltung für Bildung, Jugend und Familie und Ministerium für Bildung, Jugend und Sport des Landes Brandenburg 2015; GDSU 2013). Zentrale Komponenten des wissenschaftlichen Denkens sind das Wissenschaftsverständnis, das Theorieverständnis, das Experimentieren und die Dateninterpretation (Koerber et al. 2011). Für die Fähigkeit der Erkenntnisgenerierung durch Experimentieren ist das Verständnis von Ursache-Wirkungs-Beziehungen und deren Überprüfung mittels kontrollierter Experimente relevant (vgl. Variablenkontrollstrategie, VKS; Chen und Klahr 1999). Die Vermittlung dieses Inhalts in der Primarstufe stellt hohe didaktische Herausforderungen an die Lehrkräfte, insbesondere bei heterogenen Lernausgangslagen. Eine weitere Herausforderung besteht im grundsätzlich abnehmenden Interesse an naturwissenschaftlichen Schulfächern über die Schulzeit, die zusätzlich durch Geschlechtsunterschiede akzentuiert wird (Elster 2007; JahnkeKlein 2013; Jansen et al. 2013; Pollmeier et al. 2014).

Unterschiedliche didaktische Ansätze zur Vermittlung der VKS wurden in zahlreichen Studien untersucht (Übersicht in: Schwichow et al. 2016). Unberücksichtigt blieb hierbei bisher das Potenzial einer anhand außerschulischer Interessen individualisierten Vermittlung. Während in anderen Domänen, wie beispielsweise Mathematik oder Lesen, ein positiver Zusammenhang von Interesse und Lernen empirisch belegt wurde (Jansen et al. 2016; Schiefele et al. 1993; Walkington 2013), finden sich wenige Erkenntnisse zu den Effekten einer interessensbasierten Vermittlung naturwissenschaftlicher Inhalte im Primarbereich. Dabei stellt die Erforschung dieses Ansatzes eine relevante Erweiterung des bestehenden Forschungsstandes dar: Neben der Überprüfung des positiven Zusammenhangs von Interesse und Lernerfolg im Bereich des formalen wissenschaftlichen Denkens, können Erkenntnisse zu motivational-affektiven Faktoren des Interessenseinbezugs in einem vergleichsweise wenig beliebten Unterrichtsfach gewonnen werden (Krapp 1998; Möller 2014).

Hier schließt die vorliegende Studie an, indem im Rahmen einer Intervention die Effekte einer an individuellen Interessen ausgerichteten und so kontextualisierten Vermittlung der VKS auf das situationale Interesse am Lernkontext und am Lerngegenstand (Fragestellung 1) und auf den Lernerfolg (Fragestellung 2) untersucht werden. Zudem wird unter Annahme eines Mediationseffekts überprüft, ob die angenommenen positiven Effekte einer so kontextualisierten Vermittlung auf das Lernen der VKS über das situationale Interesse am Lernkontext und das situationale Interesse in der Lernsituation vermittelt werden (Fragestellung 3).

\section{Kompetenzbereich Naturwissenschaftliches Denken in der Grundschule}

Der vielperspektivische Sachunterricht der Grundschule legt durch die Berücksichtigung sozial- und kulturwissenschaftlicher, raumbezogener, naturbezogener, technischer und historischer Aspekte einen breiten Grundstein (natur-)wissenschaftlicher Bildung (GDSU 2013). Durch die Vermittlung inhaltsspezifischen Wissens zu Phänomenen der naturwissenschaftlichen Fächer Biologie, Chemie und Physik schafft der frühe Sachunterricht eine Grundlage für die inhaltliche Anschlussfähigkeit des 
naturwissenschaftlichen Fachunterrichts. Außerdem werden im Sachunterricht der Grundschule fächerübergreifend relevante Denk- und Arbeitsweisen der Naturwissenschaften vermittelt, wie das Aufstellen und systematische Überprüfen von Hypothesen zur experimentellen Erkenntnisgenerierung (SenBFJ und MBJS 2015; GDSU 2013). Diese zentrale Arbeitsweise ist dem Bereich des formalen wissenschaftlichen Denkens (im Gegensatz zum inhaltsbezogenen wissenschaftlichen Denken) zuzuordnen (Sodian und Mayer 2013). Eine Reihe an Studien zur Entwicklung des wissenschaftlichen Denkens von Kindern im Vor- und Grundschulalter belegen, dass bereits Kinder im Vorschulalter über grundlegende Fähigkeiten der Erkenntnisgenerierung verfügen (Bullock und Sodian 2003; Koerber 2006; Koerber et al. 2011, 2015; Sodian et al. 2002; Zimmerman 2007). Ein zentraler Befund der LOGIKStudie (Longitudinal Study on the Development of Individual Competencies) zeigt, dass Grundschülerinnen und -schüler bis zu einem Alter von zehn Jahren zwar noch erhebliche Probleme haben, ein schlüssiges experimentelles Design selbst zu planen und zu entwerfen $(<20 \%)$, aber schon $60 \%$ der Neunjährigen mit didaktischer Unterstützung ein kontrolliertes Experiment auswählen und korrekt begründen können (Bullock und Sodian 2003; s. auch Mayer et al. 2014).

Um die Fähigkeiten des formalen wissenschaftlichen Denkens systematisch zu untersuchen, wurden auf Basis eines übergreifenden Kompetenzmodells des wissenschaftlichen Denkens (Bybee 1997) differenzierte Kompetenzmodelle zum Wissenschaftsverständnis und zum Experimentieren entwickelt (Koerber et al. 2008). Auch die VKS als zentrale Fähigkeit des formalen wissenschaftlichen Denkens ist in entsprechenden Kompetenzmodellen abgebildet (Hammann 2004; Koerber et al. 2011).

\subsection{Die Variablenkontrollstrategie}

Die VKS dient der empirischen Überprüfung von Ursache-Wirkungsbeziehungen durch gezielte Parametervariation (kontrolliertes Experiment) und ist somit das grundlegende Prinzip der experimentellen Erkenntnisgenerierung (Chen und Klahr 1999). Kern der VKS ist die Variation einer interessierenden Variable unter Konstanthalten aller anderen Variablen. Nur unter Anwendung dieser Strategie kann die Auswirkung der Variation einer unabhängigen Variable auf eine abhängige Variable überprüft werden.

Nach Chen und Klahr (1999) beinhaltet das Verständnis der VKS vier Teilkomponenten: Das (1) Unterscheiden von konfundierten und unkonfundierten Experimenten, das (2) Planen von unkonfundierten Experimenten sowie das (3) Interpretieren der Ergebnisse von unkonfundierten Experimenten und das (4) Verständnis, dass Ergebnisse konfundierter Experimente keine Aussagekraft besitzen. Diese Teilkomponenten werden auch in verschiedenen Kompetenzentwicklungsmodellen zum Experimentieren berücksichtigt (z. B. Hammann 2004; Koerber et al. 2011). Koerber et al. (2011) untersuchten die Entwicklung der Experimentierfähigkeit im Grundschulalter basierend auf einer angenommenen Struktur mit drei Verständnisniveaus. Hier werden sogenannte naive Vorstellungen als unterstes Niveau angesehen, welche sich über Zwischenvorstellungen hin zu einer wissenschaftlichen Vorstellung entwickeln. In Bezug auf die VKS bedeutet dies, dass auf der untersten Stufe keine 
Variable systematisch verändert wird. Auf der Zwischenstufe wird zwar die fokale Variable manipuliert, doch die anderen Variablen nicht konstant gehalten. Die Variation der fokalen Variable unter Konstanthalten der anderen Variablen entspricht letztlich einer wissenschaftlichen Vorstellung des Experimentierens.

\subsection{Vermittlung der Variablenkontrollstrategie}

Empirische Befunde zeigen, dass die korrekte Anwendung der VKS ohne instruktionale Unterstützung über die Grundschule hinaus auch in der Adoleszenz noch schwerfällt (Zimmerman 2007).

Die Metaanalysen von Ross (1988) und Schwichow et al. (2016) belegen, dass das Verständnis der VKS durch Interventionen verbessert werden kann. Die Studien weisen Gesamteffektstärken von $g=0,73$ (Ross 1988) und $g=0,61$ (Schwichow et al. 2016) auf. Als wirksame didaktische Unterstützung zeigt sich in der Metaanalyse von Schwichow et al. (2016) die Erzeugung eines kognitiven Konflikts sowie die korrekte Demonstration von Experimenten. Den Befunden von Spiro et al. (1992) hinsichtlich der Verwendung multipler Kontexte bei der Vermittlung von Lerninhalten entsprechend, weisen die Ergebnisse der Metaanalyse von Ross (1988) ebenfalls darauf hin, dass sich das Heranziehen unterschiedlicher Aufgabenkontexte (vs. ein gleichbleibender Aufgabenkontext) und außerschulische Aufgabenkontexte (vs. schulische Aufgabenkontexte) positiv auf den Lernerfolg auswirken können. Die Dimension der Aufgabenkontexte wird in der Metaanalyse von Schwichow et al. (2016) ebenfalls anhand schulischer (z.B. Reproduktion von Bakterien) und außerschulischer (z. B. Auswirkungen gesunden Essens auf den Körper) Kontexte analysiert. Der Befund von Ross (1988) kann hier allerdings nicht vollständig repliziert werden (Schwichow et al. 2016).

Obwohl außerschulische Kontexte mit einer erhöhten subjektiven Bedeutsamkeit für den Lernenden assoziiert sein können (siehe Relevanzforschung: Albrecht und Karabenick 2018; Priniski et al. 2018; Stuckey et al. 2013) bleibt unklar, ob ein einzelner, spezifischer Kontext in einer heterogenen Lerngruppe bei allen Schülerinnen und Schülern gleichermaßen die gewünschten motivational-affektiven Prozesse auslöst, die sich mutmaßlich positiv auf das Lernen auswirken. Eine zielgerichtete individuelle Wahl von Kontexten zur Einbettung des Lerninhalts steigert vermutlich die Chance, dass Schülerinnen und Schüler von diesem Ansatz profitieren. Dieser didaktische Ansatz entspricht einer Kontextualisierung von Lernaufgaben gemäß individueller Interessensgegenstände.

\section{Interesse und Lernen}

Der Person-Gegenstands-Konzeption des Interesses (Krapp 1992; Prenzel et al. 1986; Schiefele 1991) folgend, ist Interesse stets auf einen spezifischen Gegenstand bezogen. Ein solcher Interessensgegenstand ist nicht ausschließlich als ein physisches Objekt zu verstehen, sondern umfasst ebenfalls Themen oder Tätigkeiten. Interesse als psychologisches Konstrukt zeichnet sich durch kognitive, emotionale und wertbezogene Anteile aus (Krapp und Prenzel 2011; Renninger und Hidi 
2016). Interesse ist demnach mit Vorwissen, positiven Emotionen und einem hohen Maß subjektiver Wertschätzung verbunden. Zudem erfolgt durch die mit dem Interessensgegenstand assoziierte „epistemologische Orientierung“ (Prenzel 1988) eine selbstveranlasste, wiederholte Auseinandersetzung, welche auf eine Erweiterung des Wissens über den Interessensgegenstand ausgerichtet ist (Prenzel 1988). In der Definition von Deci und Ryan (1993) wird intrinsisch motiviertes Verhalten als interessensbestimmtes Handeln beschrieben, was auf den großen Überlappungsbereich des (intrinsischen) Motivationskonstrukts mit dem Interessenkonstrukt verweist. Ähnlich definiert Krapp (1999, 2010b) Interesse als eine besondere Form der Lernmotivation. Die mit der Interessenshandlung verbundenen positiven Emotionen und die hohe subjektive Bedeutsamkeit begünstigen eine hohe ,intrinsische Qualität der Lernhandlung" (Krapp 1999).

Auf theoretischer Ebene werden zudem das individuelle und das situationale Interesse voneinander unterschieden (Hidi und Renninger 2006; Krapp 1992; Krapp und Prenzel 2011; Renninger und Hidi 2016). Während individuelle Interessen sich durch zeitlich überdauernde Person-Gegenstands-Beziehungen auszeichnen, wird situationales Interesse unmittelbar durch einen (Lern-)Gegenstand ausgelöst und ist zeitlich begrenzt. Falls dieser Gegenstand Bezüge zum individuellen Interesse aufweist, wird auch von aktualisiertem individuellen Interesse gesprochen. Ein gesteigertes situationales Interesse ist mit erhöhter Aufmerksamkeit und erhöhtem Engagement verbunden (Hidi und Renninger 2006; Krapp 2010a; Prenzel et al. 1986). Zudem wird situationalem Interesse eine entscheidende Rolle in der Genese individueller Interessen zugeschrieben (Hidi und Renninger 2006).

Diese Aspekte von Interesse legen nahe, dass es eine bedeutsame Ressource bei Lernprozessen darstellen kann. Verschiedene Autoren (Ainley et al. 2002; Hidi 1990; Krapp et al. 1992; Schiefele 1996; Wild und Schiefele 1994) bilden die Wirkweise des Interesses auf das Lernen in einem Mediationsmodell ab (Übersicht in: Krapp 2010a). Dabei wird (individuelles) Interesse als unabhängige Variable und (domänenspezifisches) Lernen als abhängige Variable beschrieben. Die mit dem situationalen Interesse in der Lernsituation verbundenen motivationalen Merkmale mediieren in dieser Vorstellung die gefundenen Effekte auf den Lernerfolg. Entsprechend dazu konnte ein grundsätzlich positiver Zusammenhang von Interesse und Lernen in verschiedenen Domänen empirisch belegt werden (Jansen et al. 2016; Schiefele et al. 1993; Walkington 2013) ohne dass die Studien dabei durchgehend ein Mediationsmodell zugrunde legten (Bernacki und Walkington 2018; Desch et al. 2016; Tröbst et al. 2016; Schiefele 1991).

\subsection{Berücksichtigung der individuellen Interessen durch Kontextualisierung}

Ein didaktischer Ansatz, welcher die lernförderlichen Eigenschaften von Interesse berücksichtigt, ist die Kontextualisierung von Lerninhalten. Diese didaktische Strategie nimmt in den naturwissenschaftlichen Fächern durch den inhärenten Bezug zu alltäglichen Phänomenen (siehe z.B.: SenBFJ und MBJS 2015) und dem Wunsch, dem Interessenabfall an mathematisch-naturwissenschaftlichen Fächern durch eine lebensweltnahe Vermittlung von Lerninhalten entgegen zu wirken (Habig 2017; Krapp 1998; Pollmeier et al. 2014; Tröbst et al. 2016), eine bedeutsame Rolle ein 
(Parchmann et al. 2008). Das Einbetten von Lerninhalten in schülernahe Erfahrungen, Alltagsphänomene oder Interessen soll die persönliche Relevanz des Themas und das Interesse am Lerngegenstand verstärken sowie das mit dem Interessensgegenstand verbundene Vorwissen aktivieren (Lohrmann 2014).

Bei der Umsetzung einer Kontextualisierung können verschiedene Kontextdimensionen berücksichtigt werden (Gilbert 2006; van Vorst et al. 2015; Walkington und Hayata 2017). Walkington und Hayata (2017) fokussieren in ihrem Ansatz das Kontextualisieren von Lernaufgaben in individuelle Interessen von Schülerinnen und Schülern. Anhand der drei instruktionspsychologischen Gestaltungsmerkmale Tiefe (depths), Spezifität des Themas (grain size) und Schülerinnen- und Schülereinbezug (ownership) kategorisieren Walkington und Hayata (2017) die Gestaltung einer interessensbasierten Aufgabenkontextualisierung. Eine mittlere bis hohe Qualität der instruktionspsychologischen Merkmalsausprägungen erweisen sich als günstige Bedingungen für Lernprozesse (Walkington und Hayata 2017). Diese berücksichtigt eine hohe Passung zwischen dem Lerninhalt und dem gewählten Kontext unter Berücksichtigung der möglichen tiefen Bezüge zu Interessen und Lebenswelt der Schülerinnen und Schüler sowie die Auswahl eines spezifischen Interessensgegenstands und der Einbezug der Schülerinnen und Schüler in Auswahl bzw. Umsetzung der Aufgabenkontextualisierung. Eine Kontextualisierung hingegen, in welcher nur oberflächliche Merkmale (z. B. Namen) berücksichtigt werden oder im Gegensatz zu einem spezifischen Interessensgegenstand (z. B. Fußball) nur ein sehr breitgefasstes Interesse (z. B. Sport) herangezogen wird, zeigt in aktuellen Studien keine positiven Effekte auf den Lernerfolg (siehe z. B.: Cakir und Simsek 2010; Høgheim und Reber 2015). Zugleich kann eine stark angereicherte Kontextualisierung den Lernerfolg negativ beeinflussen, da seductive details (für das Lösen der Aufgabe irrelevante Informationen, wie Illustrationen oder zusätzlicher Text), vom eigentlichen Lernziel ablenken und die Informationsverarbeitung beeinflussen (Harp und Mayer 1998).

Die Vielzahl an graduellen Unterschieden in der Umsetzung einer interessensbasierten Aufgabenkontextualisierung spiegelt sich in einem relativ uneinheitlichen Forschungsstand wider (Übersicht in: Walkington und Hayata 2017; Walkington und Bernacki 2020). Darüber hinaus weist der Forschungsstand zur Kontextualisierung von Lerninhalten Lücken auf (Sevian et al. 2018). Anstatt eine Kontextualisierung in Form von spezifischen Interessensgegenständen umzusetzen und dadurch die Individualisierung von Lernprozessen $\mathrm{zu}$ ermöglichen, werden in den vorliegenden Studien häufig lebensweltliche Kontexte (z.B. Kontextualisierung mittels Umgebungsmerkmalen wie Städte- oder Sportstadionnamen) bzw. unspezifische Interessensgegenstände (z.B. Kontextualisierung anhand des breitgefassten Interesses Sport anstatt einer spezifischen Sportart) zur Kontextualisierung herangezogen (Walkington und Hayata 2017). Die Ergebnisse der Metaanalyse von Schwichow et al. (2016) sowie der Überblick über relevante Studien von Walkington und Hayata (2017) zeigen, dass das Heranziehen außerschulischer Kontexte nicht automatisch lernförderliche Prozesse auslöst. Vielmehr kann angenommen werden, dass es das jeweilige Interesse an spezifischen Kontexten ist, welches sich positiv auf das Lernen auswirkt. Außerdem fokussieren die vorliegenden Studien den Mathematik- sowie den naturwissenschaftlichen Fachunterricht und damit einhergehend Schülerinnen und Schüler ab der siebten Jahrgangsstufe (Bernacki und Walkington 2018; Fancsali 
und Ritter 2014; Høgheim und Reber 2015; Kölbach und Sumfleth 2013; Ültay und Calik 2012). Insbesondere für das Grundschulalter fehlt es an Studien, die die Effekte einer in individuelle, außerschulische Interessen kontextualisierte Vermittlung von naturwissenschaftlichen Lerninhalten untersuchen.

\section{Aktuelle Studie}

Die aktuelle Studie hat zum Ziel, Kontextualisierungseffekte einer interessensbasierten Vermittlung in einem Inhaltsbereich des formalen wissenschaftlichen Denkens (VKS) zu überprüfen. Hier knüpft die Studie an bestehende Erkenntnisse zum positiven Zusammenhang von Interesse und Lernen an (Jansen et al. 2016; Schiefele et al. 1993; Walkington 2013) und erweitert diese um die Gruppe der Grundschülerinnen und Grundschüler.

Dabei wird untersucht, ob eine an individuellen Interessen ausgerichtete Vermittlung der VKS positive Effekte auf (1) das situationale Interesse am Lernkontext und am Lerngegenstand VKS und (2) auf den Lernerfolg hat. Außerdem wird unter Annahme eines Mediationseffekts die Frage adressiert, (3) ob positive Effekte einer an individuellen Interessen ausgerichtete Vermittlung auf das Lernen der VKS über das situationale Interesse am Lernkontext (= aktualisiertes individuelles Interesse) und das situationale Interesse in der Lernsituation vermittelt werden.

In Anlehnung an theoretische Modelle und empirische Ergebnisse aus der Interessensforschung erwarten wir, dass eine individualisierte Vermittlung der VKS der Vermittlung in einem Standardkontext hinsichtlich der Ausprägung des situationalen Interesses als auch des Lernerfolgs überlegen ist. Zudem nehmen wir an, dass der positive Effekt einer an individuellen Interessen ausgerichteten und so kontextualisierten Vermittlung auf den Lernerfolg über das aktualisierte Interesse am Lernkontext und über das situationale Interesse am Lerngegenstand (VKS) mediiert wird.

\section{Methode}

\subsection{Studiendesign}

Zur Beantwortung der Fragestellungen wurde eine experimentelle Interventionsstudie mit Prä- und Posttests an sechs Grundschulen in Berlin und Brandenburg durchgeführt. Zunächst wurden mit einem computergestützten Fragebogen die individuellen Interessen und das Vorwissen zur VKS der teilnehmenden Schülerinnen und Schüler erhoben. Auf Grundlage der angegebenen Interessen wurden die Schülerinnen und Schüler in Kleingruppen zu durchschnittlich vier Kindern eingeteilt (Erläuterung der Zuordnung unter Abschn. 5.3). Die Experimentalgruppe 1 (EG 1) erhielt eine entsprechend ihrer individuellen Interessen kontextualisierte Lerneinheit. Die Experimentalgruppe 2 (EG 2) erhielt eine Vermittlung der VKS in einem Standardkontext (Thema: Magnetismus), einem typischen Inhalt des naturwissenschaftlichen Sachunterrichts der Grundschule. Eine Kontrollgruppe (KG) erhielt im 
relevanten Zeitraum keine Förderung. Nach Abschluss der beiden Lerneinheiten wurde mit einem zweiwöchigen Abstand zum Prätest der Posttest durchgeführt. Zudem wurden in der Studie die Drittvariablen sprachliche Fähigkeiten (ELFE II, Lenhard et al. 2018), kognitive Grundfähigkeiten (AGTB 5-12, Hasselhorn et al. 2012; CFT20-R, Weiß 2006) sowie ein Indikator für den sozioökonomischen Status (zuhause verfügbare Bücher) erfasst.

\subsection{Stichprobe}

An der Studie haben im Zeitraum von Oktober 2018-Mai 2019 N=195 Grundschulkinder der 3. und 4. Jahrgangsstufe teilgenommen. Die Dropoutquote lag bei $16 \%$, sodass bei den folgenden Analysen $N=162$ (46\% weiblich) Schülerinnen und Schüler berücksichtigt wurden. Der Dropout erfolgte unsystematisch aufgrund krankheitsbedingter oder schulpraktischer Abwesenheit der Kinder über den Verlauf der Studie, was unterschiedliche Gruppengrößen verursachte. Die Schülerinnen und Schüler waren durchschnittlich $M=8,9$ Jahre, $S D=0,76$ alt. Die Gruppen setzten sich aus $n=89$ in der EG 1 (40\% weiblich, $M=8,9$ Jahre, $S D=0,67), n=46(54 \%$ weiblich, $M=8,9$ Jahre, $S D=0,96)$ in der EG 2 und $n=27$ (48\% weiblich, $M=8,9$; $S D=0,75)$ in der KG zusammen.

\subsection{Durchführung}

Die Studie wurde während der regulären Unterrichtszeit von geschulten Testleiterinnen und Testleitern (Studierende des Lehramts oder der Psychologie) umgesetzt. Für die EG 1 und EG 2 erstreckte sich der Studienablauf auf insgesamt fünf Treffen zu je 50 min. Die Kinder der KG erhielten keine Intervention, sondern besuchten in diesem Zeitraum den regulär stattfindenden Unterricht. Demnach erstreckte sich der Studienablauf der KG auf drei Treffen. Das erste Treffen im Klassenverband diente dazu, zunächst das Projekt und den Projektablauf vorzustellen und darauffolgend das Vorwissen zum Lerngegenstand VKS anhand eines Prätests (Edelsbrunner et al. 2018) sowie daran anschließend die kognitiven Grundfähigkeiten anhand des ersten Teils des CFT-20-R (Weiß 2006) zu erfassen. Nach durchschnittlich vier Tagen fand ein zweites Treffen in Form von Einzelsitzungen statt. Zum Einstieg initiierten die Testleiterinnen und Testleiter ein kurzes Gespräch mit dem jeweiligen Kind, in welchem die soziodemographischen Daten des Kindes mit einem kurzen Fragebogen erfasst wurden. Daran anschließend wurde die Arbeitsgedächtniskapazität anhand ausgewählter Subtests der computerbasierten Arbeitsgedächtnistestbatterie 5-12 (AGTB 5-12; Hasselhorn et al. 2012) und das individuelle Interesse anhand eines computerbasierten, eigenentwickelten Fragebogens erfasst. Auf Basis der Ratings einer Reihe von Interessensgegenständen mittels jeweils drei Items auf einer vierstufigen Skala wurden die individuellen Interessen der Kinder identifiziert.

Es folgte eine randomisierte Zuordnung der Kinder zur EG 1, EG 2 oder KG, wobei Kinder in der EG 1 mit gleichen Interessen zu Kleingruppen zusammengefasst wurden. Als Kriterium zur Aufnahme in eine Interessensgruppe wurde ein eher stark ausgeprägtes Interesse $(M \geq 3)$ festgelegt. Zudem nahmen schulpraktische sowie organisatorische Aspekte, wie bspw. die angestrebte Gruppengröße von vier 
Tab. 1 Überblick über die Erhebungs- und Interventionseinheiten

\begin{tabular}{|c|c|c|c|c|c|}
\hline & Termin 1 & Termin 2 & Termin 3 & Termin 4 & Termin 5 \\
\hline \multirow[t]{3}{*}{$\begin{array}{l}\text { Erge- } \\
\text { bungs- } \\
\text { instru- } \\
\text { mente }\end{array}$} & $\begin{array}{l}\text { Prätest zum } \\
\text { Verständnis } \\
\text { der VKS } \\
(\mathrm{MZP} 1)\end{array}$ & $\begin{array}{l}\text { Fragebogen } \\
\text { Soziodemo- } \\
\text { graphische } \\
\text { Daten }\end{array}$ & $\begin{array}{l}\text { Interventionssitzung } 1 \\
\text { (IS1) zur Ver- } \\
\text { mittlung der } \\
\text { VKS }\end{array}$ & $\begin{array}{l}\text { Interventionssitzung } 2 \\
\text { (IS 2) zur Ver- } \\
\text { mittlung der } \\
\text { VKS }\end{array}$ & $\begin{array}{l}\text { Posttest } \\
\text { zum Ver- } \\
\text { ständnis } \\
\text { der VKS } \\
\text { (MZP2) }\end{array}$ \\
\hline & CFT-20-R & AGTB 5-12 & $\begin{array}{l}\text { Fragebogen Situa- } \\
\text { tionales Interesse } \\
\text { an Lernkontext und } \\
\text { Lerngegenstand }\end{array}$ & $\begin{array}{l}\text { Fragebogen Situa- } \\
\text { tionales Interesse } \\
\text { an Lernkontext und } \\
\text { Lerngegenstand }\end{array}$ & - \\
\hline & - & $\begin{array}{l}\text { Fragebogen } \\
\text { Erhebung } \\
\text { individueller } \\
\text { Interessen }\end{array}$ & ELFE II & - & - \\
\hline Gruppen & $\begin{array}{l}\text { EG 1, } \\
\text { EG 2, KG }\end{array}$ & $\begin{array}{l}\text { EG 1, EG 2, } \\
\mathrm{KG}\end{array}$ & EG 1, EG 2 & EG 1, EG 2 & $\begin{array}{l}\text { EG 1, } \\
\text { EG 2, KG }\end{array}$ \\
\hline
\end{tabular}

Kindern, Einfluss auf die Gruppenzuordnung. Es resultierten insgesamt 27 Kleingruppen in der EG 1 (neun zum Thema Fußball, sechs zum Thema Lesen, vier zum Thema Schwimmen, drei zum Thema Reiten, drei zum Thema Turnen und zwei zum Thema Fahrradfahren) und 11 Kleingruppen in der EG 2 zum Thema Magnetismus.

Das dritte und vierte Treffen bildeten die beiden Interventionssitzungen (IS) die mit einem zeitlichen Abstand von durchschnittlich vier Tagen durchgeführt wurden. Neben der Vermittlung des Lerninhalts wurde nach beiden IS das situationale Interesse am Lernkontext und am Lerngegenstand (angelehnt an: Ainley et al. 2002) sowie das Textverständnis anhand eines Subtests der ELFE II (Lenhard et al. 2018) erhoben. Bei einem letzten Treffen wurde erneut das Verständnis der VKS anhand eines Posttests (Edelsbrunner et al. 2018) im Klassenverband erhoben (vgl. Tab. 1).

Die Instruktionen sowie die einzelnen Items des Tests zur Erfassung des Verständnisses der VKS im Prä- und Posttest und des Fragebogens zur Erfassung des situationalen Interesses am Interventionsinhalt sowie zum individuellen Interesse wurden von den Testleiterinnen und Testleitern vorgelesen, um Effekte der Lesekompetenz weitgehend zu reduzieren. Die verwendeten Instrumente werden im nächsten Kapitel beschrieben.

\subsection{Instrumente}

\subsubsection{Erfassung des Verständnisses der VKS}

Zur Prüfung des Verständnisses der VKS im Prä- und Posttest wurde ein Test von Edelsbrunner et al. (2018) herangezogen. Der Test enthält eine Reihe etablierter Aufgaben zur VKS. Um die Durchführungsdauer zu reduzieren und Überschneidungen von thematischen Items (,Tiere“ und „Spiel“) mit den Interessensbereichen der IS zu vermeiden, wurden von den insgesamt vierzehn Testitems neun Items $(7 \times$ Multiple Choice mit drei Antwortmöglichkeiten, $2 \times$ offenes Antwortformat) ausgewählt. Diese gekürzte Version des Testverfahrens wies eine eingeschränkte interne Konsistenz 
(Cronbachs $\alpha$ ) von 0,64 auf, die in Teilen mit den recht unterschiedlichen Aufgabenformaten erklärbar ist. Jede richtige Antwort der Multiple-Choice-Aufgaben wurde mit einem, jede Bewertungsaufgabe mit offenem Antwortformat mit maximal zwei Punkten bepunktet. Somit summierte sich die Maximalpunktzahl auf 11 Punkte. Die offenen Antworten wurden von zwei unabhängigen Ratern ausgewertet, die Interraterreliabilität Cohens Kappa betrug 0,81. Korrelationsanalysen zeigten, dass ein hoher positiver Zusammenhang zwischen der Prätest- und der Posttestleistung $(r=0,48 ; p<0,001)$ besteht. Neben der Auswertung der Rohwerte wurden die Antwortmuster angelehnt an die Kompetenzstufen nach Koerber et al. (2011) ausgewertet. Fünf von neun Aufgaben erfassten das Verständnis der korrekten Variablenkontrolle (im Gegensatz zum Verständnis der korrekten Variablenvariation). Die Antwortmöglichkeiten dieser fünf Multiple-Choice-Aufgaben waren auf Basis einer naiven Vorstellung, einer Zwischenvorstellung oder einer wissenschaftlichen Vorstellung formuliert. Wurden mindestens vier von fünf Antworten auf einer Niveaustufe gegeben, wird dies als ein konsistentes Antwortmuster dieser Niveaustufe gewertet.

\subsubsection{Instrument zur Erhebung der individuellen Interessen}

Angelehnt an die Ergebnisse relevanter Studien (z. B. Pruisken 2005; Wolfert und Pupeter 2018) sowie einer von uns durchgeführten Vorstudie zu individuellen Interessen von Kindern im Grundschulalter wurde ein computerbasierter, adaptiver Fragebogen entwickelt. Den Kindern wurden dabei am Laptop zunächst sechs übergeordnete Themenbereiche (Sport, Musik, Ruhige Beschäftigungen, Tiere, Technik, Spielen) präsentiert und zur (Mehrfach-)Auswahl angeboten. In Abhängigkeit der individuellen Ratings wurden adaptiv zu den ausgewählten Themenbereichen im Durchschnitt fünf spezifische Interessensgegenstände (z. B. Themenbereich Sport: Fußballspielen, Basketballspielen, Handballspielen, Reiten, Fahrradfahren, Schwimmen, Turnen) präsentiert. Die Kinder bewerteten ihr Interesse an dem jeweiligen Interessensgegenstand auf einer anhand von Gesichtern visualisierten Skala mit vier Abstufungen $(1-\odot, 4-())$. Im Anschluss daran wurden für die als interessant bewerteten Interessensgegenstände (Ausprägung $\geq 3$ ) die kognitive (,Ich weiß schon viel über das Thema XY."), die affektive (,Wenn ich mich mit dem Thema XY beschäftige, vergesse ich alles um mich herum. “) und die wertbezogene („Das Thema XY ist mir persönlich wichtig. ") Komponente des individuellen Interesses auf der zuvor genutzten Skala erhoben (vgl. Ainley et al. 2002). Der Fragebogen wurde durch ein offenes Antwortformat zu weiteren Interessensgegenständen ergänzt.

\subsubsection{Instrument zur Erhebung des situationalen Interesses am Lernkontext und am Lerngegenstand VKS}

Zur Erfassung des wahrgenommenen situationalen Interesses an dem dargebotenen Lernkontext und dem Lerngegenstand VKS wurde ein Fragebogen mit zwei Skalen und je sechs Items im vierstufigen Likert-Format $(1=$ ich stimme gar nicht $\mathrm{zu}$ bis $4=$ ich stimme sehr stark zu) verwendet (angelehnt an: Ainley et al. 2002). Es wurde die kognitive (z.B.: „Über das Thema Fußball weiß ich schon ziemlich viel.“), die 
affektive (z. B.: „Es macht mir Spaß, mich mit dem Thema Fußball zu beschäftigen. “) und die wertbezogene (z. B.: „,Das Thema Fußball ist mir persönlich wichtig. “) Komponente des Interesses am Kontextthema berücksichtigt. Das situationale Interesse am Lerngegenstand VKS wurde ebenfalls entsprechend der drei Komponenten erhoben: kognitiv (z.B.: „Ich könnte jemandem erklären, was ein fairer Vergleich ist“ (kindgerechte Formulierung für kontrolliertes Experiment)), affektiv (z. B.: ,Es hat mir Spaß gemacht mich mit fairen Vergleichen zu beschäftigen.") und wertbezogen (z.B.: „Was ich über faire Vergleiche gelernt habe, finde ich persönlich wichtig.“). Die interne Konsistenz (Cronbachs $\alpha$ ) der Skalen Kontext $(0,87)$ und Lerngegenstand $(0,79)$ sowie die Retest-Reliabilitäten der Skalen Lernkontext $(r=0,82)$ und Lerngegenstand $(r=0,70)$ sind als gut bzw. akzeptabel zu bewerten.

\subsubsection{Interventionsmaterial und Vermittlung der VKS}

Ziel der Intervention war es, Grundschulkindern die VKS als grundlegende Experimentierstrategie zu vermitteln, sodass sie bei einem Experiment mit mehreren Variablen kausale Wirkzusammenhänge schlüssig identifizieren und beschreiben können. Dazu wurde ein Interventionsleitfaden sowie Interventionsmaterialien (angelehnt an: Edelsbrunner et al. 2018) entwickelt und in mehreren Vorversuchen geprüft und angepasst. Die Intervention umfasste zwei Lerneinheiten mit einem Umfang von jeweils ca. 50 min. Die Sitzungen enthielten die Punkte (1) Begrüßung, (2) Aufgreifen der Interessen aus dem Vortest, (3) Einführung in die Problemstellung mit Arbeitsblatt, (4) Erklärung von Vergleichen (allgemein), (5) Erklärung von „,fairen“ Vergleichen, (6) gemeinsames Anwenden und Üben von „fairen“ Vergleichen am Beispiel 1, (7) gemeinsames Anwenden und Üben am Beispiel 2, (8) Ergebnissicherung.

Nach der Begrüßung und der Einbettung der Interessen aus dem Vortest $(1,2)$ wurde auf dem Arbeitsblatt (3) durch eine kurze Cover-Story auf das individuelle Interesse der Schülerinnen und Schüler Bezug genommen und eine fiktive Meinungsverschiedenheit zwischen zwei Kindern dargestellt, welche als Ausgangspunkt zur Beschäftigung mit fairen Vergleichen diente. Bei einer entsprechend des Interessensgegenstands Fußball kontextualisierten Aufgabe war die Leitfrage z. B. ,Woran liegt es, ob ein Fußball beim Abschlag weit oder weniger weit fliegt?“. In einer geleiteten Gruppendiskussion wurden gemeinsam mögliche Einflussfaktoren diskutiert (z.B. Größe des Balls, Härte des Balls, Beschaffenheit des Untergrunds). Um den Protagonisten der Geschichte zu helfen, ihre Vermutung zu überprüfen (z.B. „Ich vermute, es liegt an der Härte des Balls!“) wurden nun unterschiedliche Vergleiche thematisiert. Es wurde geprüft, welche Arten von Vergleichen geeignet sein könnten, um die Vermutung zu bestätigen oder zu verwerfen. Als Erweiterung wurde in (Punkt 5) der faire Vergleich eingeführt, d.h. ein Vergleich, der nur einen Einflussfaktor fokussiert (z. B. Vergleich eines harten Balls und eines weichen Balls) und alle anderen Faktoren konstant hält (z. B. Größe des Balls, Untergrund). Die Materialien der beiden Lerneinheiten unterschieden sich insofern, dass mögliche Vergleiche (a) anhand von drei Antwortmöglichkeiten oder (b) von der graphischen Darstellung eines konfundierten und eines unkonfundierten Vergleichs diskutiert wurden. Die Aufgaben der EG 1 und EG 2 unterschieden sich nur hinsichtlich ihrer thematischen Einbettung. 
Die zugrundeliegende Aufgabenstruktur, Satzstruktur und Satzlänge waren identisch. Durch die leitfadengestützte Vermittlung konnte ein fester Ablauf gesichert werden, um die Vergleichbarkeit zwischen den Interventionsbedingungen sicherzustellen.

\subsubsection{Drittvariablen}

Um Unterschiede, bzw. den Einfluss sprachlicher Kompetenzen der Schülerinnen und Schüler kontrollieren bzw. abschätzen zu können, wurde der ELFE II-Test: Ein Leseverständnistest für Erst- bis Siebtklässler - Version II (Lenhard et al. 2018) eingesetzt. Es handelt sich dabei um ein standardisiertes Verfahren mit einer hohen Zuverlässigkeit $\left(r_{t t}=0,93\right)$. Des Weiteren wurden mit dem CFT20-R (Weiß 2006) die kognitiven Grundfähigkeiten der Kinder erfasst, um den Einfluss kognitiver Voraussetzungen auf das Verständnis der VKS kontrollieren zu können. Dieser sprachfreie standardisierte Test weist gute Kennwerte der Reliabilität auf (CFT20-R: $\left.r_{t t}=0,80\right)$. Als Proxy für den familiären sozioökonomischen Hintergrund (SES) wurde die Anzahl der Bücher zu Hause über ein bildbasiertes, fünfstufiges Rating erfasst (Stubbe et al. 2012). Mit einem Kurzfragebogen wurden wichtige demografische Informationen wie Alter, Geschlecht, sprachlicher Hintergrund und Lieblingsfächer der Kinder erfasst.

\section{Ergebnisse}

\subsection{Deskriptive Ergebnisse: Drittvariablen}

Vorabanalysen zeigten, dass es keine Unterschiede zwischen der EG 1, EG 2 und KG hinsichtlich der erfassten Drittvariablen kognitive Grundfähigkeiten, Leseverständnis und SES gibt. Dieser Eindruck wurde durch die Ergebnisse einer Varianzanalyse bestätigt (alle $p s>0,05$ ) (vgl. Tab. 2), sodass die Drittvariablen in den folgenden Analysen nicht berücksichtigt werden.

Tab. 2 Deskriptive Werte $(M$ und $S D)$ zu den Drittvariablen nach Gruppen

\begin{tabular}{llllll}
\hline & EG 1 & EG 2 & KG & Gesamt & $p$ \\
\hline ELFE II $^{\text {a }}$ & $10,5(4,6)$ & $9,5(4,6)$ & $9,8(5,5)$ & $10,1(4,7)$ & 0,45 \\
& $n=88$ & $n=42$ & $n=19$ & $N=149$ & \\
CFT20-R $^{b}$ & $25,9(5,9)$ & $25,2(5,5)$ & $23,8(6,4)$ & $25,4(5,9)$ & 0,25 \\
& $n=89$ & $n=46$ & $n=27$ & $N=162$ & \\
SES $^{c}$ & $3,6(1,2)$ & $3,4(1,3)$ & $3,4(1,4)$ & $3,5(1,2)$ & 0,70 \\
& $n=87$ & $n=46$ & $n=26$ & $N=159$ & \\
\hline
\end{tabular}

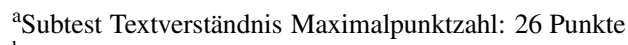

${ }^{\mathrm{b}}$ Kurzform Maximalpunktzahl: 46 Punkte

cauf 5-er Likert-Skala 


\subsection{Fragestellung 1: Hat eine an individuellen Interessen ausgerichtete Vermittlung der VKS positive Effekte auf das situationale Interesse am Lernkontext und am Lerngegenstand?}

Anhand einer einfaktoriellen ANOVA wurde zunächst untersucht, ob Lernkontexte, die auf Basis geäußerter individueller Interessen der Kinder manipuliert wurden, interessanter eingeschätzt werden als der Standardkontext Magnetismus. Darüber hinaus wurde analysiert, ob Gruppenunterschiede zwischen der EG 1 und EG 2 hinsichtlich des situationalen Interesses an dem Lerngegenstand VKS bestehen.

Überprüfung der Implementierung Zur Überprüfung der Implementierung wurde das Instrument zur Erhebung des situationalen Interesses am Lernkontext und am Lerngegenstand VKS herangezogen und zunächst hinsichtlich des Lernkontexts ausgewertet (individueller Kontext vs. Kontext Magnetismus).

Deskriptive Analysen zeigten, dass nach beiden IS die EG 1 gegenüber der EG 2 ein durchschnittlich deutlich höheres Interesse an den Lernkontexten aufweist (vgl. Tab. 3). Eine einfaktorielle ANOVA zeigte, dass dieser Unterschied zu beiden Erhebungszeitpunkten signifikant ist (IS1: $F(1,111)=45,1 ; p<0,001 ; \eta^{2}=0,29$; IS2: $\left.F(1,112)=48,8 ; p<0,001 ; \eta^{2}=0,30\right)$.

Trotz eines relativ hohen Interesses der EG 2 am Lernkontext Magnetismus, zeigte die EG 1 ein signifikant höheres Interesse an den individualisierten Lernkontexten. Dieses Ergebnis lässt auf eine erfolgreiche Implementierung der Intervention schließen.

\section{Gruppenunterschiede des situationalen Interesses am Lerngegenstand VKS}

Die EG 1 schätzte ihr Interesse am Lerngegenstand VKS gegenüber der EG 2 signifikant höher ein (vgl. Tab. 3). Eine einfaktorielle ANOVA zeigte, dass dieses durchschnittlich höhere situationale Interesse am Lerngegenstand VKS der EG 1 gegenüber der EG 2 nach beiden IS bei mittleren bis starken Effektgrößen signifikant ist $\left(F(1,105)=6,5 ; p=0,01 ; \eta^{2}=0,06\right.$; IS2: $\left.F(1,107)=22,9 ; p<0,001 ; \eta^{2}=0,18\right)$.

\subsection{Fragestellung 2: Hat eine an individuellen Interessen ausgerichtete Vermittlung der VKS positive Effekte auf den Lernerfolg?}

Um zu untersuchen, ob eine an individuellen Interessen ausgerichtete und so kontextualisierte Vermittlung der VKS positive Effekte auf den Lernerfolg hat, wurden die

Tab. 3 Interesse am Lernkontext und am Lerngegenstand VKS ( $M$ und $S D$ ) zu zwei Erhebungszeitpunkten auf 4-er Likert-Skala ${ }^{\mathrm{a}}$

\begin{tabular}{lll}
\hline & EG 1 $(n=78)$ & EG 2 $(n=36)$ \\
\hline Interesse am Lernkontext IS 1 & $3,0(0,74)$ & $2,1(0,62)$ \\
Interesse am Lernkontext IS 2 & $3,1(0,67)$ & $2,1(0,74)$ \\
Interesse am Lerngegenstand IS 1 & $3,1(0,69)$ & $2,8(0,69)$ \\
Interesse am Lerngegenstand IS 2 & $3,2(0,64)$ & $2,5(0,80)$ \\
\hline
\end{tabular}

a-er Likert Skala ( $1=$ ich stimme gar nicht zu bis $4=$ ich stimme sehr stark zu) 
Gruppenunterschiede in der Prä- und Posttestleistung anhand einer Mixed ANOVA mit anschließenden post-hoc Vergleichen analysiert.

In einer differenzierten Analyse auf Aufgabenebene (je fünf Aufgaben im Präund Posttest) wurde das konzeptuelle Verständnis der VKS zudem bezogen auf die Niveaus nach Koerber et al. (2011) zu MZP 1 und MZP 2 untersucht.

Lernzuwachs Die Mixed ANOVA zeigte einen signifikanten Haupteffekt des Faktors Zeit auf die Leistung im Posttest $(F(1,159)=102,2 ; p<0,001$; partielles $\left.\eta^{2}=0,39\right)$. Auch der Haupteffekt des Faktors Gruppe wurde signifikant ( $F(2$, $159)=5,1 ; p<0,01$; partielles $\left.\eta^{2}=0,06\right)$. Die Interaktion der beiden Faktoren Zeit und Gruppe zeigte ebenfalls einen signifikanten Effekt $(F(2,159)=4,2 ; p=0,02$; partielles $\left.\eta^{2}=0,05\right)$.

Anschließende post-hoc Tests (Tukey-HSD) zeigten keinen Unterschied in der Prätestleistung zwischen EG 1 und EG 2 (MDiff =0,5; $p=0,44 ; g=0,2)$. Auch die KG wies keine signifikanten Unterschiede der Prätestleistung zur EG 1 (MDiff =-0,7; $p=0,33 ; g=0,3)$ und zur EG 2 (MDiff $=-0,2 ; p=0,93 ; g=0,1)$ auf. Außerdem zeigten die post-hoc Tests keinen Unterschied in der Posttestleistung zwischen EG 1 und EG $2(M D i f f=-0,6 ; p=0,53 ; g=0,2)$. Gegenüber der KG erreichte sowohl die EG 1 $(M D i f f=2,4 ; p=0,001 ; g=0,8)$ als auch die EG $2(M D i f f=1,8 ; p=0,03 ; g=0,6)$ eine signifikant bessere Posttestleistung (vgl. Abb. 1).

Es gab einen statistisch signifikanten Effekt der Zeit für alle drei Gruppen: EG 1 $\left(F(1,88)=108,8 ; p<0,001\right.$; partielles $\left.\eta^{2}=0,55\right)$, EG $2(F(1,45)=53,9 ; p<0,001$; partielles $\left.\eta^{2}=0,55\right)$, KG: $\left(F(1,26)=6,43 ; p=0,02\right.$; partielles $\left.\eta^{2}=0,20\right)$. Die Ergebnisse der Analysen legen nahe, dass die EG 1 und die EG 2 einen ähnlichen Lernzuwachs im Verständnis der VKS über die Zeit verzeichnen, bei einem sehr geringen deskriptiven Vorteil der EG 1 gegenüber der EG 2. Die KG verzeichnet einen geringen signifikanten Lernzuwachs über die Zeit und unterscheidet sich in der Posttestleistung signifikant von der EG 1 und der EG 2.

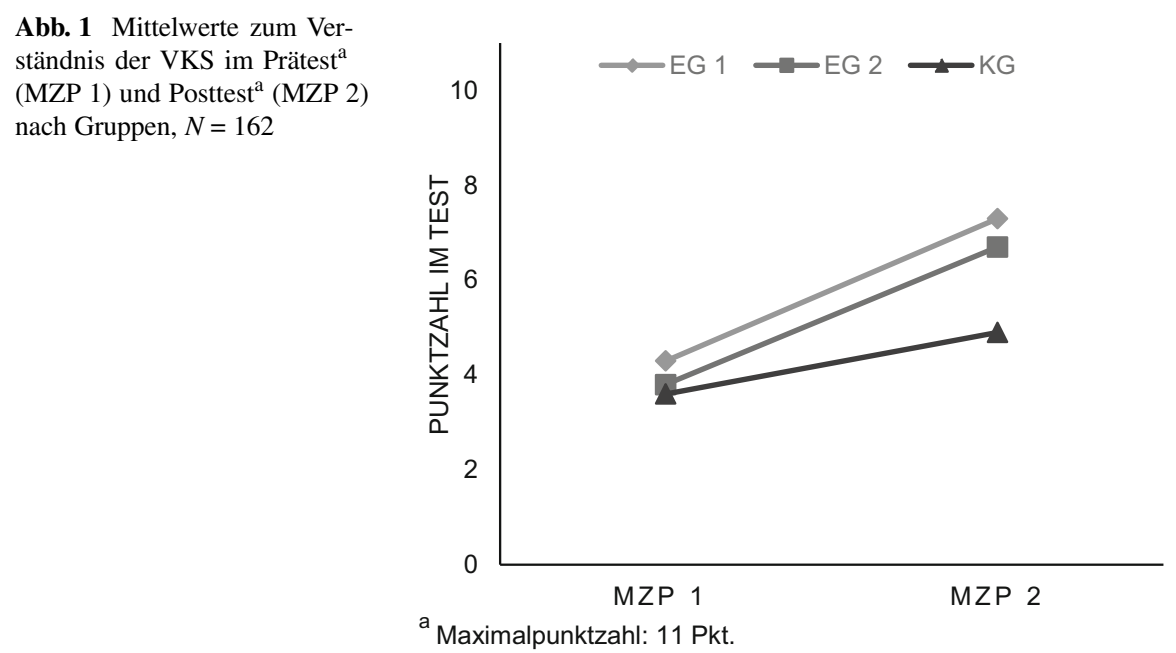


Lernzuwachs bezogen auf Verständnisniveaus der VKS Angelehnt an Koerber et al. (2011) werden in der folgenden Analyse die Häufigkeiten der Antworten zu Aufgaben der korrekten Variablenkontrolle im Prä- bzw. Posttest auf den jeweiligen Verständnisniveaus betrachtet. Werden mindestens vier von fünf Antworten auf einem Niveau gegeben, wird dies als ein konsistentes Antwortmuster dieses Verständnisniveaus gewertet.

Die graphische Darstellung (vgl. Abb. 2 und 3) der Antwortmuster nach Gruppen zu MZP 1 und MZP 2 zeigt folgenden Trend: In allen drei Gruppen wiesen zu MZP 1 über $60 \%$ der Kinder ein unspezifisches Antwortmuster auf, indem sich die gewählten Antworten nicht konsistent einem Verständnisniveau zuordnen lassen. $21 \%$ der Kinder in der EG 1, 14\% der Kinder in der EG 2 und 15\% der Kinder in der KG zeigten ein Antwortmuster auf wissenschaftlichem Niveau.

Zur Überprüfung von Unterschieden zwischen den Gruppen wurde der exakte Fisher-Test durchgeführt, da manche erwarteten Zellhäufigkeiten kleiner als 5 waren. Zu MZP 1 gab es keine signifikanten Gruppenunterschiede der Antwortmuster nach Verständnisniveau (Naive Vorstellung: $\mathrm{x}^{2}(2)=2,61 ; p=0,34 ; V=0,13$; Zwischenvorstellung: $\mathrm{x}^{2}(2)=5,43 ; p=0,06 ; V=0,17$; Wissenschaftliche Vorstellung: $\mathrm{X}^{2}(2)=1,04 ; p=0,61, V=0,09$; Unspezifisch.: $\left.\mathrm{x}^{2}(2)=4,04 ; p=0,13, V=0,16\right)$.

$\mathrm{Zu}$ MZP 2 zeigten $56 \%$ der Kinder der KG weiterhin ein unspezifisches Antwortmuster und hinzukommend weitere $4 \%$ der Kinder der KG ein Antwortmuster

Abb. 2 Gestapeltes Balkendiagramm zum Antwortmuster nach Gruppen zu MZP 1, Angaben in Prozent, $N=162$

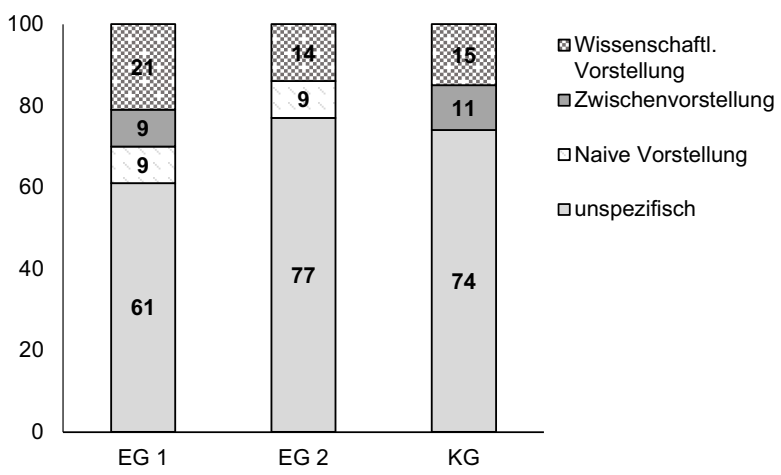

Abb. 3 Gestapeltes Balkendiagramm zum Antwortmuster nach Gruppen zu MZP 2, Angaben in Prozent, $N=162$

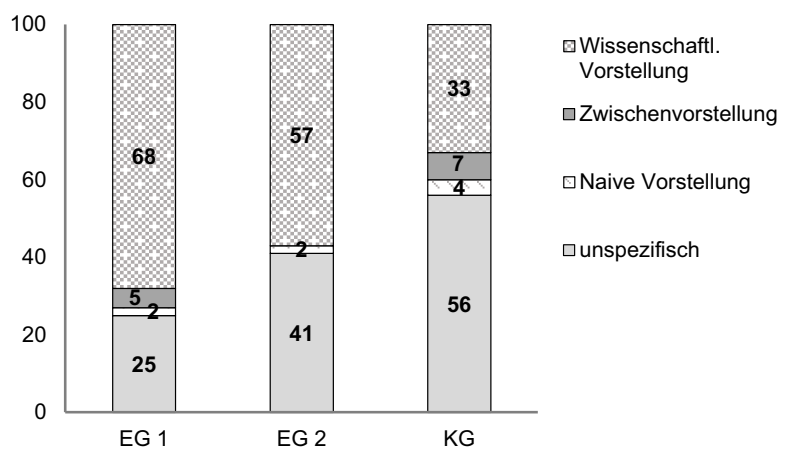


auf dem Niveau einer naiven Vorstellung. In der EG 1 (68\%) und der EG 2 (57\%) erreichte hingegen die Mehrheit der Kinder ein konsistentes Antwortmuster auf dem Niveau einer wissenschaftlichen Vorstellung (vgl. Abb. 3).

Es zeigten sich signifikante Unterschiede zwischen den Gruppen hinsichtlich der Antwortmuster auf dem Verständnisniveau einer wissenschaftlichen Vorstellung $\left(\mathrm{x}^{2}(2)=10,35 ; p=0,006 ; V=0,26\right)$ und der unspezifischen Antwortmuster $\left(\mathrm{x}^{2}(2)=9,59 ; p=0,008 ; V=0,25\right)$. Für das Niveau der Zwischenvorstellung $\left(\mathrm{x}^{2}(2)=3,04 ; p=0,20 ; V=0,14\right)$ und der naiven Vorstellung $\left(\mathrm{x}^{2}(2)=0,72 ; p=0,81\right.$; $V=0,04)$ zeigten sich keine signifikanten Gruppenunterschiede.

Das konzeptuelle Verständnis der VKS auf Niveau einer wissenschaftlichen Vorstellung ist von MZP 1 zu MZP 2 in der EG 1 um 47\% und in der EG 2 um $43 \%$ angestiegen. Dies zeigt einen geringen deskriptiven Vorteil der EG 1 gegenüber der EG 2 und einen signifikanten Vorteil der EG 1 und der EG 2 gegenüber der KG $(+8 \%)$.

\subsection{Fragestellung 3: Werden positive Effekte einer an individuellen Interessen ausgerichteten Vermittlung auf das Lernen der VKS über das situationale Interesse am Lernkontext und das situationale Interesse in der Lernsituation mediiert?}

Die Annahme eines seriellen Mediationseffekts wurde mittels des Moduls PROCESS (Hayes 2018) im Statistikprogramm SPSS geprüft.

Mediationsanalyse Die Mediationsanalyse mit PROCESS (Hayes 2018) basiert auf linearen Regressionen nach der Methode der kleinsten Quadrate zur Ermittlung der unstandardisierten Pfadkoeffizienten. Bootstrapping mit 10,000 Iterationen wurde eingesetzt, um Konfidenzintervalle und Inferenzstatistiken zu berechnen.

Die Effekte wurden als signifikant erachtet, wenn das Konfidenzintervall nicht Null einschloss. Anhand der seriellen Mediationsanalyse wurde überprüft, ob der Effekt der Interventionsbedingung (Codierung: EG 2=1, EG 1=2) auf die Posttestleistung durch das aktualisierte Interesse am Lernkontext und das situationale Interesse am Lerngegenstand VKS vermittelt wird. Die Reihenfolge der Mediatoren ergibt sich auf Grundlage theoretischer und empirischer Befunde zur Steigerung des situationalen Interesses in der Lernsituation durch interessensbasierte Kontextualisierung (Ainley et al. 2002).

Der totale Effekt der Interventionsbedingung auf die Posttestleistung wurde nicht signifikant $(B=0,98 ; p=0,11)$. Die Interventionsbedingung sagte das aktualisierte Interesse am Lernkontext signifikant vorher $(B=0,97 ; p<0,001)$, welches wiederum das situationale Interesse am Lerngegenstand signifikant vorhersagte $(B=0,62$; $p<0,001)$. Das situationale Interesse am Lerngegenstand sagte wiederum die Posttestleistung signifikant vorher $(B=1,3 ; p=0,01)$. Außerdem zeigte sich ein negativer Effekt des aktualisierten Interesses am Lernkontext auf die Posttestleistung $(B=-1,4$; $p<0,01)$. Die Interventionsbedingung stellte keinen signifikanten Prädiktor für das situationale Interesse am Lerngegenstand dar $(B=0,05 ; p=0,75)$ (vgl. Abb. 4).

Der positive indirekte Effekt über das aktualisierte Interesse am Lernkontext und das situationale Interesse am Lerngegenstand wurde signifikant (indirekter Effekt 


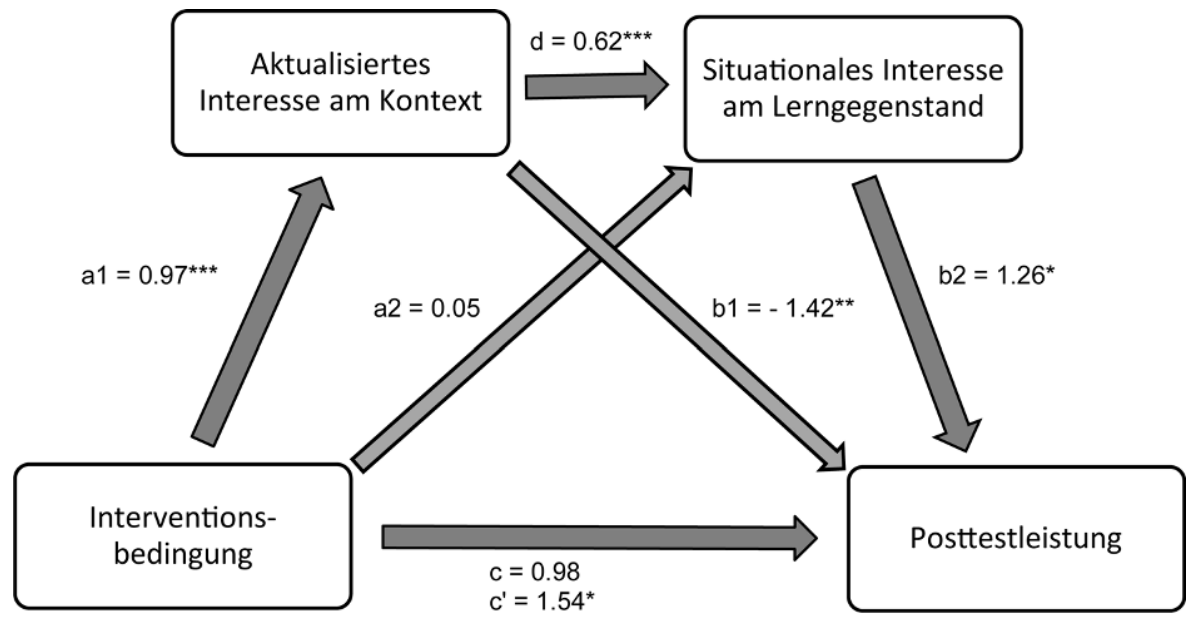

${ }^{\star} p<.05,{ }^{* *} p<.01,{ }^{* * *} p<.001$

Abb. 4 Angenommenes Mediationsmodell, $N=108$

$\mathrm{a} 1 \times \mathrm{d} \times \mathrm{b} 2=0,25 ; 95 \%$-CI $[0,05 ; 0,47])$. Der negative indirekte Effekt der Interventionsbedingung über das Interesse am Lernkontext auf die Posttestleistung wurde ebenfalls signifikant (indirekter Effekt a $1 \times \mathrm{b} 1=-0,46 ; 95 \%$-CI $[-0,82 ;-0,16]$ ). Der indirekte Effekt der Interventionsbedingung über das situationale Interesse am Lerngegenstand wurde nicht signifikant $(\mathrm{a} 2 \times \mathrm{b} 2=0,02 ; 95 \%$-CI $[-0,09 ; 0,15])$. Unter Berücksichtigung der indirekten Effekte wurde der direkte Effekt der Interventionsbedingung auf die Posttestleistung signifikant $(B=1,54 ; p=0,02)$. Die unterschiedlichen Vorzeichen der indirekten Effekte weisen auf einen Supressoreffekt hin.

\section{Diskussion}

Die vorliegende Studie untersuchte die Effekte einer an individuellen Interessen ausgerichteten und so kontextualisierten Vermittlung auf das situationale Interesse am Lernkontext und am Lerngegenstand (Fragestellung 1) und den Lernerfolg (Fragestellung 2). Zudem wurde der Zusammenhang einer interessensbasierten Vermittlung und des Lernerfolgs unter Annahme eines Mediationseffekts überprüft (Fragestellung 3). Als Lerngegenstand fungierte die VKS, ein lehrplanrelevanter Inhalt des naturwissenschaftlichen Sachunterrichts. Zur Beantwortung der Fragestellungen wurde eine experimentelle Interventionsstudie mit Prä- und Posttests durchgeführt. Die Analysen unterstützen die Annahme positiver Effekte einer interessensbasierten Vermittlung des Lerngegenstands auf das situationale Interesse von Grundschülerinnen und -schülern. Zwar zeigte sich die Intervention zur Vermittlung der VKS grundsätzlich als effektiv, der Lernerfolg im Verständnis der VKS konnte durch die experimentelle Manipulation jedoch nicht signifikant gesteigert werden. In einer Mediationsanalyse wurde die Wirkrichtung der untersuchten Konstrukte geprüft. Auf Basis der vorhandenen Daten zeigten sich entgegengesetzte Effekte: Einerseits 
konnte die Annahme, dass der positive Zusammenhang der Interventionsbedingung und des Lernerfolgs über das aktualisierte Interesse am Lernkontext und das situationale Interesse am Lerngegenstand mediiert wird, untermauert werden. Andererseits zeigte sich ein negativer indirekter Effekt der Interventionsbedingung über das aktualisierte Interesse am Lernkontext auf den Lernerfolg.

Fragestellung 1 Hat eine an individuellen Interessen ausgerichtete Vermittlung der VKS positive Effekte auf das situationale Interesse am Lernkontext und am Lerngegenstand?

Anhand der Analyse des Interesses am Lernkontext kann davon ausgegangen werden, dass die Implementierung der Intervention erfolgreich war und die Unterschiede im angegebenen situationalen Interesse am Lerngegenstand der EG 1 und der EG 2 auf die individualisierten Kontexte zurückzuführen sind. Die dargestellten Ergebnisse der Intervention stehen im Einklang mit empirischen Befunden zu Steigerung des situationalen Interesses an einem Lerngegenstand durch eine Aufgabenkontextualisierung (Bernacki und Walkington 2018; Hidi und Renninger 2006; Høgheim und Reber 2015; Kölbach und Sumfleth 2013). Trotz eines ebenfalls relativ hohen Interesses der EG 2 am Kontext Magnetismus zeigte die EG 1 ein signifikant höheres Interesse sowohl an den individualisierten Lernkontexten als auch am Lerngegenstand VKS. Es wurde bei der Auswahl des Kontexts Magnetismus in der Kontrollbedingung bewusst darauf verzichtet, den Lernkontext der EG 2 künstlich unattraktiv zu gestalten. Durch vielfache Alltagserfahrungen (z. B. Spielzeuge, Küchenmagnete) werden Präkonzepte zum Thema Magnetismus bereits im Kindergarten- und Vorschulalter entwickelt, die häufig mit einer hohen Faszination für dieses Thema einhergehen (Jeschonek 2012). Dass der Vergleich des Interesses an einem fachbezogenen und gleichzeitig alltagsnahen Kontext (Magnetismus) mit dem Interesse an individualisierten Kontexten dennoch zugunsten der EG 1 ausfällt, unterstreicht die positive Wirkung der individualisierten Kontexte auf das situationale Interesse in der Lernsituation und erhöht zudem die ökologische Validität der Befunde.

Eine Erweiterung der Erkenntnisse ist in der vorliegenden Studie durch die explizite Operationalisierung des situationalen Interesses am Lernkontext und am Lerngegenstand zu sehen. Die Wirkweise des situationalen Interesses kann dadurch differenziert abgebildet werden und trägt somit zum besseren Verständnis der zugrundeliegenden Mechanismen bei.

Fragestellung 2 Hat eine an individuellen Interessen ausgerichtete Vermittlung der VKS positive Effekte auf den Lernerfolg?

Die signifikante Interaktion Zeit $\times$ Gruppe deutet zunächst auf die grundsätzliche Wirksamkeit der Intervention hinsichtlich des Verständnisses der VKS hin. Offensichtlich entwickeln sich die Gruppen im Verständnis der VKS über die Zeit unterschiedlich. Die anschließenden Analysen zeigen jedoch, dass sowohl die EG 1 als auch die EG 2 von der Intervention ähnlich profitierten und ein durchschlagender Effekt der Individualisierung nicht zu beobachten ist. Trotz eines geringen deskriptiven Vorteils der EG 1 gegenüber der EG 2 bestehen signifikante Unterschiede der EG 1 nur gegenüber der KG. Auch für die KG zeigte sich ein signifikanter Effekt des Fak- 
tors Zeit auf das Verständnis der VKS. Als mögliche Erklärung können Testeffekte oder Effekte von Treatmentdiffusion (beispielsweise in Form von Pausengesprächen über Inhalte der Interventionssitzungen) herangezogen werden.

Die Ergebnisse stimmen mit den ergänzenden Analysen zu den Verständnisniveaus der VKS überein. Ausgehend von typischen, mehrheitlich naiven Verständnismustern oder Mustern auf dem Niveau von Zwischenvorstellungen (Bullock und Sodian 2003; Koerber et al. 2011) zeigen EG 1 und EG 2 vergleichbare positive Entwicklungen über die Zeit. Im Vergleich zur KG sind in diesen Gruppen nach der Intervention deutlich häufiger wissenschaftlich adäquate Vorstellungen beobachtbar. Die Ergebnisse decken sich zum einen mit Befunden zum Verständnis der VKS im Grundschulalter ohne didaktische Unterstützung, zum anderen mit Befunden zur grundsätzlichen Einflussnahme auf das Verständnis der VKS durch zielgerichtete didaktische Interventionen (Bullock und Sodian 2003; Ross 1988; Schwichow et al. 2016).

Zur Beantwortung der Fragestellung 2 sind neben den signifikanten Unterschieden in der Posttestleistung der EG 1 und EG 2 gegenüber der KG insbesondere die nicht signifikanten Unterschiede der EG 1 und der EG 2 erklärungsbedürftig. In den beiden IS erhielt sowohl die EG 1 als auch die EG 2 gezielte didaktische Unterstützung beim Erlernen der VKS (vgl. Interventionsmaterial und Vermittlung der $V K S$ ). Die jeweils kleinschrittige, strukturierte Vermittlung des Lerngegenstands mit grundsätzlich ansprechenden Lernkontexten in beiden Gruppen stellt eine plausible Erklärung für den geringen deskriptiven Vorteil der EG 1 gegenüber der EG 2 hinsichtlich des globalen Testwerts und des operationalisierten konzeptuellen Verständnisses der VKS dar (vgl. z. B.: Chen und Klahr 1999; Klahr und Nigam 2004).

Fragestellung 3 Werden positive Effekte einer an individuellen Interessen ausgerichteten Vermittlung auf das Lernen der VKS über das situationale Interesse am Lernkontext und das situationale Interesse in der Lernsituation mediiert?

Theoretischen Annahmen folgend wurde ein serielles Mediationsmodell spezifiziert und geprüft. Der nicht signifikante totale Effekt der Interventionsbedingung auf die Posttestleistung stellt nach den theoretischen Überlegungen von Zhao et al. (2010) keine Einschränkung für die Annahme eines Mediationseffekts dar. Die Ergebnisse der Mediationsanalyse weisen auf einen Supressoreffekt hin: Wird durch die interessensbasierte Interventionsbedingung (EG 1) ausschließlich ein hohes Interesse am Kontext der Vermittlung (jedoch nicht am Lerngegenstand) aktiviert, wirkt sich dies negativ auf die Posttestleistung aus. Als plausible Interpretation kann hier die ablenkende Wirkung einer interessensbasierten Vermittlung (vgl. seductive detail effect, Harp und Mayer 1998) herangezogen werden. Wird jedoch das Interesse am Lerngegenstand durch das Interesse am Lernkontext aktiviert, zeigt sich in Übereinstimmung mit Bernacki und Walkington (2018) hypothesenkonform ein positiver indirekter Effekt des situationalen Interesses auf die Posttestleistung. Sowohl der Einfluss der Lernmaterialien als auch ablenkende Effekte sollten in zukünftigen Studien für die Zielgruppe der Grundschülerinnen und -schüler gezielt untersucht werden. 


\subsection{Limitationen}

Gerade in experimentellen Feldstudien treten zwangläufig Bedingungen auf, die die Interpretation der Ergebnisse einschränken. Als zentrale Limitation sind die unterschiedlichen Gruppengrößen der EG 1, EG 2 und KG zu nennen. Zwar wurden grundsätzlich entsprechend angepasste statistische Verfahren herangezogen, jedoch schränken die teilweise geringen Stichprobengrößen die Testpower ein. Die Schätzungen sind daher als konservativ anzusehen. Ebenfalls aufgrund geringer Stichprobengrößen wurde auf Mehrebenenanalysen verzichtet, für die eine angemessene Fallzahl sicherlich unterschritten worden wäre. Zudem wurden durch die randomisierte Zuordnung der Kinder zu den Interventionsbedingungen keine „natürlichen“ Gruppen untersucht, wodurch das Nichtberücksichtigen einer genesteten Datenstruktur in den Analysen weniger problematisch erscheint. Die instruktionspsychologischen Gestaltungsmerkmale nach Walkington und Hayata (2017) wurden zwar bei der Entwicklung der Lernmaterialien berücksichtigt, allerdings in der Umsetzung zum Teil nicht ausreichend kontrolliert. Die Spezifität des Themas (grain size) konnte durch die Erhebung der Interessen individuell passend manipuliert und durch das Instrument zur Erhebung des situationalen Interesses am Lernkontext und am Lerngegenstand kontrolliert werden. Der Schülerinnen- und Schülereinbezug (ownership) wurde ebenfalls durch die Erhebung individueller Interessen umgesetzt und in der EG 1 durch den Verweis auf die eigene Themenwahl den Lernenden explizit gemacht. Inwiefern die Schülerinnen und Schüler sich in den Gestaltungsprozess der Kontextualisierung einbezogen fühlten und inwiefern sie die Kontextualisierung als authentisch in Bezug auf ihren Interessensgegenstand (depths) wahrnahmen, wurde nicht erhoben. Als didaktischer Mechanismus sollte gerade die Wahrnehmung von Wahlautonomie (vgl. Deci und Ryan 1993) in zukünftigen Studien erfasst und ggf. gezielt manipuliert werden, da sie sich als wirksam z. B. für das situationale Interesse herausgestellt hat (vgl. Desch et al. 2016).

Um längerfristige Entwicklungen abbilden zu können, sind zudem verzögerte follow-up Tests sinnvoll. Solche Tests wurden im Rahmen der Studie geplant und durchgeführt. Aufgrund von deutlichen Einschränkungen im Rücklauf konnten diese Daten jedoch nicht umfassend ausgewertet werden.

\subsection{Implikationen}

Trotz zahlreicher empirischer Belege zum positiven Zusammenhang von Interesse und Lernen und der Verankerung von Interesse als relevanter Anknüpfungspunkt für die Inhaltsvermittlung in den Rahmenlehrplänen (z. B. SenBFJ und MBJS 2015) zeigten Studien, dass in der alltäglichen Unterrichtsgestaltung die individuellen Interessen der Kinder nur ungenügend beachtet werden (Hössl 2006; Pannek et al. 2015). Dabei ist durch die hohe Heterogenität der Lern- und Entwicklungsstände in der Grundschule (Vock und Gronostaj 2017) der Bedarf zur Entwicklung von individualisieren Unterrichtsmethoden hoch. Die Interessensvielfalt von Grundschülerinnen und -schülern (Pruisken 2005) kann dabei als Ressource verstanden werden, um adaptive Zugänge zu einem Lerngegenstand durch eine interessensbasierte Unterrichtsgestaltung zu eröffnen. Vor dem Hintergrund theoretischer Überlegungen 
zur Bedeutung von Interesse in Lernprozessen (Deci und Ryan 1993; Krapp 2010b) bekräftigen die dargestellten affektiv-motivationalen Effekte des Interessenseinbezugs das hohe Potenzial einer interessensbasierten Unterrichtsgestaltung insbesondere für vergleichsweise unbeliebte Unterrichtsfächer. Um die Realisierung einer interessensbasierten Unterrichtsgestaltung zu forcieren, müssen Hürden auf Seiten der Lehrkräfte, wie eine zeitaufwendige Umsetzung (Pannek et al. 2015) identifiziert und abgebaut werden. Neben dem Einsatz von intelligenten tutoriellen Systemen (vgl. z. B. Walkington 2013) kann eine lehrergesteuerte Aufgabenkontextualisierung mit Bezug zu typischen Schülerinnen- und Schülerinteressen in Kombination mit der individuellen Schülerinnen- und Schülerauswahl der angebotenen Kontexte eine praktikable, zeiteinsparende Umsetzung darstellen.

\subsection{Ausblick}

Um das Potenzial individualisierender Unterrichtsansätze auf Basis außerschulischer Interessen weiter zu untersuchen, sind differenzielle Analysen des Zusammenhangs von Interesse und Lernen am Lerngegenstand VKS geplant. Unter Berücksichtigung von unterschiedlichen Lernausgangslagen der Kinder kann bspw. untersucht werden, ob Kinder mit schwachen kognitiven oder sprachlichen Lernvoraussetzungen von einem individualisierten Vorgehen in besonderer Weise profitieren oder aber von ablenkenden Details stärker negativ beeinflusst werden. Die Identifikation weiterer konzeptueller Lerninhalte für die Umsetzung einer interessensbasierten Individualisierung stellt einen weiteren Schritt dar. Neben der inhaltlichen Erweiterung um weitere Lerngegenstände sollten die instruktionspsychologischen Gestaltungsmerkmale bei der Individualisierung verstärkt untersucht werden. Perspektivisch können so die didaktischen Grundlagen geschaffen werden, für zentrale unterrichtliche Inhalte individualisierend auf die Voraussetzungen einer kognitiv und sprachlich heterogenen Schülerschaft eingehen zu können.

Funding This research was funded by the DFG, award number KE 2196/1-1. Open Access funding enabled and organized by Projekt DEAL.

Open Access Dieser Artikel wird unter der Creative Commons Namensnennung 4.0 International Lizenz veröffentlicht, welche die Nutzung, Vervielfältigung, Bearbeitung, Verbreitung und Wiedergabe in jeglichem Medium und Format erlaubt, sofern Sie den/die ursprünglichen Autor(en) und die Quelle ordnungsgemäß nennen, einen Link zur Creative Commons Lizenz beifügen und angeben, ob Änderungen vorgenommen wurden.

Die in diesem Artikel enthaltenen Bilder und sonstiges Drittmaterial unterliegen ebenfalls der genannten Creative Commons Lizenz, sofern sich aus der Abbildungslegende nichts anderes ergibt. Sofern das betreffende Material nicht unter der genannten Creative Commons Lizenz steht und die betreffende Handlung nicht nach gesetzlichen Vorschriften erlaubt ist, ist für die oben aufgeführten Weiterverwendungen des Materials die Einwilligung des jeweiligen Rechteinhabers einzuholen.

Weitere Details zur Lizenz entnehmen Sie bitte der Lizenzinformation auf http://creativecommons.org/ licenses/by/4.0/deed.de. 


\section{Literatur}

Ainley, M., Hidi, S., \& Berndorff, D. (2002). Interest, learning, and the psychological processes that mediate their relationship. Journal of Educational Psychology, 94(3), 545-561.

Albrecht, J.R., \& Karabenick, S. A. (2018). Relevance for learning and motivation in education. Journal of Experimental Education, 86(1), 1-10.

Berliner Senatsverwaltung für Bildung, Jugend und Familie \& Ministerium für Bildung, Jugend und Sport des Landes Brandenburg (2015). Rahmenlehrplan für die Jahrgangsstufen 1-10 der Berliner und Brandenburger Schulen. https://bildungsserver.berlin-brandenburg.de/unterricht/rahmenlehrplaene/ implementierung-des-neuen-rahmenlehrplans-fuer-die-jahrgangsstufen-1-10/amtliche-fassung. Zugegriffen: 27. Dez. 2020.

Bernacki, M. L., \& Walkington, C. (2018). The role of situational interest in personalized learning. Journal of Educational Psychology, 110(6), 864-881.

Bullock, M., \& Sodian, B. (2003). Entwicklung des wissenschaftlichen Denkens. In W. Schneider \& M. Knopf (Hrsg.), Entwicklung, Lehren und Lernen (S. 75-92). Göttingen: Hogrefe.

Bybee, R.W. (1997). Toward an understanding of scientific literacy. In W. Gräber \& C. Bolte (Hrsg.), Scientific literacy: an international symposium (S. 37-68). Kiel: IPN.

Cakir, O., \& Simsek, N. (2010). A comparative analysis of the effects of computer and paper-based personalization on student achievement. Computers \& Education, 55(4), 1524-1531.

Chen, Z., \& Klahr, D. (1999). All other things being equal. Acquisition and transfer of the control of variables strategy. Child Development, 70(5), 1098-1120.

Deci, E.L., \& Ryan, R.M. (1993). Die Selbstbestimmungstheorie der Motivation und ihre Bedeutung für die Pädagogik. Zeitschrift für Pädagogik, 39(2), 223-238.

Desch, I., Stiller, C., \& Wilde, M. (2016). Förderung des situationsspezifischen Interesses durch eine Schülerwahl des Unterrichtsthemas. Psychologie in Erziehung und Unterricht, 1, 60-74.

Edelsbrunner, P.A., Schalk, L., Schumacher, R., \& Stern, E. (2018). Variable control and conceptual change. A large-scale quantitative study in elementary school. Learning and Individual Differences, $66,38-53$.

Elster, D. (2007). In welchen Kontexten sind naturwissenschaftliche Inhalte für Jugendliche interessant? Ergebnisse der ROSE-Erhebung in Österreich und Deutschland. Plus Lucis, 3, 2-8.

Fancsali, S.E., \& Ritter, S. (2014). Context personalization, preferences, and performance in an intelligent tutoring system for middle school mathematics. In M. Pistilli, J. Willis, D. Koch, K. Arnold, S. Teasley \& A. Pardo (Hrsg.), Proceedings of the Fourth International Conference on Learning Analytics And Knowledge. LAK '14. (S. 73-77). New York: ACM.

Fischer, F., Kollar, I., Ufer, S., Sodian, B., Hussmann, H., Pekrun, R., Neuhaus, B., Dorner, B., Pankofer, S., Fischer, M., Strijbos, J.-W., Heene, M., \& Eberle, J. (2014). Scientific reasoning and argumentation. Advancing an interdisciplinary research agenda in education. Frontline Learning Research, 5 , $28-45$.

GDSU (2013). Perspektivrahmen Sachunterricht (vollständig erw. und überarb. Auflage). Bad Heilbrunn: Klinkhardt.

Gilbert, J. K. (2006). On the nature of "context" in chemical education. International Journal of Science Education, 28(9), 957-976.

Habig, S. (2017). Studien zum Physik- und Chemielernen. Systematisch varriierte Kontextaufgaben und ihr Einfluss auf kognitive und affektive Schülerfaktoren. Berlin: Logos.

Hammann, M. (2004). Kompetenzentwicklungsmodelle. Merkmale und ihre Bedeutung dargestellt anhand von Kompetenzen beim Experimentieren. Der mathematische und naturwissenschaftliche Unterricht, 57(4), 196-203.

Harp, S.F., \& Mayer, R.E. (1998). How seductive details do their damage. Journal of Educational Psychology, 90(3), 414-434.

Hasselhorn, M., Schumann-Hengsteler, R., Gronauer, J., Grube, D., Mähler, C., Schmid, I., Seitz-Stein, K., \& Zoelch, C. (2012). AGTB 5-12 Arbeitsgedächtnistestbatterie für Kinder von 5 bis 12 Jahren. Göttingen: Hogrefe.

Hayes, A.F. (2018). Introduction to mediation, moderation, and conditional process analysis: a regressionbased approach (2. Aufl.). New York: Guilford.

Hidi, S. (1990). Interest and its contribution as a mental resource for learning. Review of Educational Research, 60(4), 549-571.

Hidi, S., \& Renninger, K. A. (2006). The four-phase model of interest development. Educational Psychologist, 41(2), 111-127. 
Høgheim, S., \& Reber, R. (2015). Supporting interest of middle school students in mathematics through context personalization and example choice. Contemporary Educational Psychology, 42, 17-25.

Hössl, A. (2006). Strategien zwischen Anpassung und Verweigerung. Die Grundschule aus der Sicht der Kinder. Diskurs Kindheits- und Jugendforschung, 1(4), 481-495.

Jahnke-Klein, S. (2013). Benötigen wir eine geschlechtsspezifische Pädagogik in den MINT-Fächern? Ein Überblick über die Debatte und den Forschungsstand. Schulpädagogik heute, 4(8), 1-19.

Jansen, M., Lüdtke, O., \& Schroeders, U. (2016). Evidence for a positive relation between interest and achievement. Examining between-person and within-person variation in five domains. Contemporary Educational Psychology, 46, 116-127.

Jansen, M. C., Schroeders, U., \& Stanat, P. (2013). Motivationale Schülermerkmale in Mathematik und den Naturwissenschaften. In H. A. Pant, P. Stanat, U. Schroeders, A. Roppelt, T. Siegle \& C. Pöhlmann (Hrsg.), IQB-Ländervergleich 2012. Mathematische und naturwissenschaftliche Kompetenzen am Ende der Sekundarstufe I (S. 348-365). Münster: Waxmann.

Jeschonek, S. (2012). Entwicklung des Verständnisses des Phänomens Magnetismus bei Kindern im Alter von 3 bis 6 Jahren. In Stiftung Haus der kleinen Forscher (Hrsg.), Wissenschaftliche Untersuchungen zur Arbeit der Stiftung „Haus der kleinen Forscher“ (S. 69-83). Schaffhausen: SCHUBI.

Klahr, D., \& Nigam, M. (2004). The equivalence of learning paths in early science instruction. Effects of direct instruction and discovery learning. Psychological Science, 15(10), 661-667.

Koerber, S. (2006). Entwicklung des wissenschaftlichen Denkens bei Vier- bis Achtjährigen. Beiträge zur Lehrerbildung, 24(2), 192-201.

Koerber, S., Mayer, D., Osterhaus, C., Schwippert, K., \& Sodian, B. (2015). The development of scientific thinking in elementary school. A comprehensive inventory. Child Development, 86(1), 327-336.

Koerber, S., Sodian, B., Kropf, N., Mayer, D., \& Schwippert, K. (2011). Die Entwicklung des wissenschaftlichen Denkens im Grundschulalter. Theorieverständnis, Experimentierstrategien, Dateninterpretation. Zeitschrift für Entwicklungspsychologie und Pädagogische Psychologie, 43(1), 16-21.

Koerber, S., Sodian, B., Thoermer, C., \& Grygier, P. (2008). Wissen über Wissenschaft als Teil der frühen naturwissenschaftlichen Bildung. In H. Giest, A. Hartinger \& J. Kahlert (Hrsg.), Kompetenzniveaus im Sachunterricht. Forschungen zur Didaktik des Sachunterrichts, (Bd. 7, S. 135-154). Bad Heilbrunn: Klinkhardt.

Kölbach, E., \& Sumfleth, E. (2013). Analyse von Kontexteffekten beim Lernen mit Lösungsbeispielen im Fach Chemie. Zeitschrift für Didaktik der Naturwissenschaften, 19, 159-188.

Krapp, A. (1992). Interesse, Lernen, Leistung. Neuere Ansätze der pädagogisch-psychologischen Interessenforschung. Arbeiten zur sozialwissenschaftlichen Psychologie, Bd. 26. Münster: Aschendorff.

Krapp, A. (1998). Entwicklung und Förderung von Interessen im Unterricht. Psychologie in Erziehung und Unterricht, 44, 185-201.

Krapp, A. (1999). Intrinsische Lernmotivation und Interesse. Forschungsansätze und konzeptuelle Überlegungen. Zeitschrift für Pädagogik, 45(3), 387-406.

Krapp, A. (2010a). Interesse. In D. H. Rost (Hrsg.), Handwörterbuch Pädagogische Psychologie 4. Aufl. Weinheim: Beltz.

Krapp, A. (2010b). Die Bedeutung von Interessen für Lernmotivation und das schulische Lernen. Eine Einführung. In I. Hemmer \& M. Hemmer (Hrsg.), Schülerinteressen an Themen, Regionen und Arbeitsweisen des Geographieunterrichts. Ergebnisse der empirischen Forschung und deren Konsequenzen für die Unterrichtspraxis. Geographiedidaktische Forschungen, (Bd. 46, S. 9-26). Weingarten: HGD.

Krapp, A., \& Prenzel, M. (2011). Research on Interest in Science. Theories, methods, and findings. International Journal of Science Education, 33(1), 27-50.

Krapp, A., Hidi, S., \& Renninger, K. A. (1992). Interest, learning, and development. In K. A. Renninger, S. Hidi \& A. Krapp (Hrsg.), The role of interest in learning and development (S. 3-25). Hillsade: Lawrence Erlbaum.

Lenhard, W., Lenhard, A., \& Schneider, W. (2018). ELFE II. Ein Leseverständnistest für Erst- bis Siebtklässler Version II. Göttingen: Hogrefe.

Lohrmann, K. (2014). Kontextualisierung und Dekontextualisierung. In W. Einsiedler (Hrsg.), Handbuch der Grundschulpädagogik und Grundschuldidaktik (4. Aufl. S. 414-419). Bad Heilbrunn: Klinkhardt.

Mayer, D., Sodian, B., Koerber, S., \& Schwippert, K. (2014). Scientific reasoning in elementary school children. Assessment and relations with cognitive abilities. Learning and Instruction, 29, 43-55.

Möller, K. (2014). Vom naturwissenschaftlichen Sachunterricht zum Fachunterricht. Der Übergang von der Grundschule in die weiterführende Schule. Zeitschrift für Didaktik der Naturwissenschaften, 20(1), $33-43$. 
Pannek, M., Schubert, L. M., Thoms, S., \& Lichtblau, M. (2015). „,... wenn die Kinder wirklich an einem Thema interessiert sind, dann lernen sie auch besser!“ - Einbezug kindlicher Interessen in den inklusiven Unterricht. Sonderpädagogische Förderung heute, 60(1), 24-37.

Parchmann, I., Ralle, B., \& Di Fuccia, D.-S. (2008). Chemie im Kontext - ein Weg zu einem anderen Chemieunterricht? In R. Demuth, C. Gräsel, I. Parchmann \& B. Ralle (Hrsg.), Chemie im Kontext. Von der Innovation zur nachhaltigen Verbreitung eines Unterrichtskonzepts (S. 9-47). Münster: Waxmann.

Pollmeier, K., Walper, L. M., Lange, K., Kleickmann, T., \& Möller, K. (2014). Vom Sachunterricht zum Fachunterricht. Physikbezogener Unterricht und Interessen im Übergang von der Primar- zu Sekundarstufe. Zeitschrift für Grundschulforschung, 7(2), 129-145.

Prenzel, M. (1988). Die Wirkweise von Interesse. Ein pädagogisch-psychologisches Erklärungsmodell. Beiträge zur psychologischen Forschung, Bd. 13. Opladen: Westdeutscher Verlag.

Prenzel, M., Krapp, A., \& Schiefele, U. (1986). Grundzüge einer pädagogischen Interessentheorie. Zeitschrift für Pädagogik, 32(2), 163-173.

Priniski, S. J., Hecht, C. A., \& Harackiewicz, J. M. (2018). Making learning personally meaningful. A new framework for relevance research. Journal of Experimental Education, 86(1), 11-29.

Pruisken, C. (2005). Grundschüler und ihre Freizeit: Sind Kinder heute gering und einseitig interessiert? Zeitschrift für Lernforschung, 33(3), 272-288.

Renninger, K.A., \& Hidi, S.E. (2016). The power of interest for motivation and engagement. New York: Routledge.

Ross, J. A. (1988). Controlling variables. A meta-analysis of training studies. Review of Educational Research, 58(4), 405-437.

Schiefele, U. (1991). Interest, learning, and motivation. Educational Psychologist, 26(3/4), 299-323.

Schiefele, U. (1996). Topic interest, text representation, and quality of experience. Contemporary Educational Psychology, 21, 3-18.

Schiefele, U., Krapp, A., \& Schreyer, I. (1993). Metaanalyse des Zusammenhangs von Interesse und schulischer Leistung. Zeitschrift für Entwicklungspsychologie und Pädagogische Psychologie, 10(2), 120-148.

Schwichow, M. G., Croker, S., Zimmerman, C., Höffler, T. N., \& Härtig, H. (2016). Teaching the controlof-variables strategy. A meta-analysis. Developmental Review, 39, 37-63.

Sevian, H., Dori, Y. J., \& Parchmann, I. (2018). How does STEM context-based learning work: what we know and what we still do not know. International Journal of Science Education, 40(10), 1095-1107.

Sodian, B., \& Mayer, D. (2013). Entwicklung des wissenschaftlichen Denkens im Vor- und Grundschulalter. In M. Stamm \& D. Edelmann (Hrsg.), Handbuch frühkindliche Bildungsforschung (S. 617-633). Wiesbaden: Springer.

Sodian, B., Thoermer, C., Kircher, E., Grygier, P., \& Günther, J. (2002). Vermittlung von Wissenschaftsverständnis in der Grundschule. In M. Prenzel \& J. Doll (Hrsg.), Bildungsqualität von Schule. Schulische und außerschulische Bedingungen mathematischer, naturwissenschaftlicher und überfachlicher Kompetenzen. 45. Beiheft zur Zeitschrift für Pädagogik. (S. 192-206). Weinheim: Beltz.

Spiro, R. J., Feltovich, P. J., Jacobson, M. J., \& Coulson, R. L. (1992). Cognitive flexibility, constructivism, and hypertext: advanced knowledge acquisition in ill-structured domains. Educational Technology, 31(5), 24-33.

Stubbe, T.C., Tarelli, I., \& Wendt, H. (2012). Soziale Disparitäten der Schülerleistungen in Mathematik und Naturwissenschaften. In W. Bos, H. Wendt, O. Köller \& C. Selter (Hrsg.), TIMSS 2011. Mathematische und naturwissenschaftliche Kompetenzen von Grundschulkindern in Deutschland im internationalen Vergleich (S. 231-244). Münster: Waxmann.

Stuckey, M., Hofstein, A., Mamlok-Naaman, R., \& Eilks, I. (2013). The meaning of 'relevance' in science education and its implications for the science curriculum. Studies in Science Education, 49(1), 1-34.

Tröbst, S., Kleickmann, T., Lange-Schubert, K., Rothkopf, A., \& Möller, K. (2016). Instruction and students' declining interest in science. An analysis of German fourth- and sixth-grade classrooms. American Educational Research Journal, 53(1), 162-193.

Ültay, N., \& Calik, M. (2012). A thematic review of studies into the effectiveness of context-based chemistry curricula. Journal of Science Education and Technology, 21, 686-701.

Vock, M., \& Gronostaj, A. (2017). Umgang mit Heterogenität in Schule und Unterricht. Berlin: FriedrichEbert-Stiftung.

van Vorst, H., Dorschu, A., Fechner, S., Kauertz, A., Krabbe, H., \& Sumfleth, E. (2015). Charakterisierung und Strukturierung von Kontexten im naturwissenschaftlichen Unterricht. Vorschlag einer theoretischen Modellierung. Zeitschrift für Didaktik der Naturwissenschaften, 21(1), 29-39. 
Walkington, C. (2013). Using adaptive learning technologies to personalize instruction to student interests. The impact of relevant contexts on performance and learning outcomes. Journal of Educational Psychology, 105(4), 932-945.

Walkington, C., \& Bernacki, M. L. (2020). Appraising research on personalized learning: definitions, theoretical alignment, advancements, and future directions. Journal of Research on Technology in Education, 52(3), 235-252.

Walkington, C., \& Hayata, C. A. (2017). Designing learning personalized to students' interests. balancing rich experiences with mathematical goals. ZDM, 49(4), 519-530.

Weiß, R. H. (2006). Grundintelligenztest Skala 2 - Revision (CFT20-R). Göttingen: Hogrefe.

Wild, K.-P., \& Schiefele, U. (1994). Aufmerksamkeit als Mediator des Einflusses von Interesse auf die Lernleistung. Sprache \& Kognition, 13(3), 138-145.

Wolfert, S., \& Pupeter, M. (2018). Freizeit: Hobbys und Mediennutzung. In World Vision Deutschland (Hrsg.), Kinder in Deutschland 2018. 4. World Vision Kinderstudie (S. 95-125). Weinheim: Beltz.

Zachmann, K., \& Ehlers, S. (2019). Wissen und Begründen. Evidenz als umkämpfte Ressource in der Wissensgesellschaft. Einleitung. In K. Zachmann \& S. Ehlers (Hrsg.), Wissen und Begründen. Evidenz als umkämpfte Ressource in der Wissensgesellschaft (S. 9-30). Baden-Baden: Nomos.

Zhao, X., Lynch Jr., J.G., \& Chen, Q. (2010). Reconsidering Baron and Kenny: myths and truths about mediation analysis. Journal of Consumer Research, 37(2), 197-206.

Zimmerman, C. (2007). The development of scientific thinking skills in elementary and middle school. Developmental Review, 27(2), 172-223. 